\title{
Estudio contrastivo de los modelos evaluativos de aula de instituciones públicas y privadas de la ciudad de Tuluá y su relación con índices de calidad educativa
}

\section{Contrastive study of the evaluative models of classroom of public and private institutions of the city of Tulua and its relation with educational quality indices}

Gustavo Adolfo González Cruz ${ }^{1}$

Diana Katherine González ${ }^{2}$

\section{Gustavo Adolfo Cárdenas Messa ${ }^{3}$}

Unidad Central del Valle del Cauca,

Tuluá, Colombia

$1 \quad$ Académico de UCEVA. ggonzalez@uceva.edu. co Facultad de Ciencias de La Educación. https://orcid. org/0000-0002-4492-1077 . https://scholar.google.com/cita tions? user=hrmTmVOAAAAJ\&hl=es

2 Directora Oficina de Internacionalización. Uceva Office of International Affairs Director. https://orcid.org/00000002-4523-0673, GOOGLE ACADEMICO: https://scholar. google.com/citations?user=7FJXb30AAAAJ\&hl=es dkgonzalez@uceva.edu.co

3 Académico de Uceva gcardenas@uceva.edu.co. https://orcid.org/0000-0002-7571-9023

https://scholar.google.es/citations? user=VJ1r4hkAAAAJ\&hl =es

\section{RESUMEN}

La propuesta investigativa Estudio Contrastivo de los Modelos Evaluativos de los docentes de tres instituciones públicas y tres privadas de la ciudad de Tuluá y su relación con índices de calidad Educativa. Tuvo la finalidad de identificar las concepciones nacionales sobre evaluación, establecidas en la ley 115 de 1994 y sus actos 
reglamentarios, además del sistema documental del Ministerio de Educación Nacional. Esta identificación permitió de forma consistente establecer una hoja de ruta, un deber ser, frente a las directrices evaluativas de los PEI y a las prácticas evaluativas.

Igualmente pretendió identificar la naturaleza establecida en los PEI de tres instituciones de educación media pública y tres privadas, con el fin de verificar la consonancia entre éstas $y$ las directivas nacionales $y$ las diferencias $y$ similitudes entre las directrices evaluativas del sector público y privado.

Se obtuvo información que permitió dilucidar el por qué en Tuluá los resultados evaluativos de las instituciones privadas supera (en las pruebas Saber 11) a las instituciones públicas, además de si en ambos casos, se responde o no a las políticas nacionales al respecto.

\section{ABSTRACT}

The research proposal Contrastive Study of the Evaluative Models of teachers from three public and three private institutions in the city of Tuluá and its relationship with educational quality indices. It had the purpose of identifying the national conceptions on evaluation, established in Law 115 of 1994 and its regulatory acts, in addition to the documentary system of the Ministry of National Education. This identification consistently allowed establishing a roadmap, a must be, against the evaluation guidelines of the PEI and evaluative practices.

It also sought to identify the nature established in the PEls of three public and three private secondary education institutions, in order to verify the consistency between these and the national directives and the differences and similarities between the evaluative guidelines of the public and private sector.

Information was obtained that allowed us to clarify why in Tuluá the evaluative results of private institutions exceed (in the Saber 11 tests) public institutions, in addition to whether or not they respond to national policies in this regard.

KEYWORDS: conceptions, evaluative practices, Saber 11 tests, quality.

\section{INTRODUCCIÓN}

Uno de los elementos fundantes de la práctica pedagógica en la evaluación, y en los modelos tradicionales está concebida como un mecanismo de control y de clasificación de los estudiantes, en términos de buenos y malos. Los modelos contemporáneos reconocen la evaluación como un ejercicio esencial dentro del proceso de aprendizaje, como verificador de los aprendizajes de los estudiantes, y en esa medida, un mecanismo de toma de decisiones.

En el caso de esta investigación, hay varios elementos sobre los cuales no hay diagnósticos de contexto ni caracterizaciones que permitan la toma de decisiones: El primero de ellos es el ajuste de los modelos evaluativos de los PEI institucionales a las directrices del estado. El segundo es la similitud o diferencia entre los modelos evaluativos usados en la educación pública y privada. El tercero es la forma como estos modelos evaluativos inciden en los procesos de aprendizaje de los estudiantes y en sus resultados académicos (evidenciados en las pruebas Saber 11) y en los resultados institucionales (Índice sintético de calidad).

En Tuluá no existen estudios que permitan a los directivos institucionales y Secretaría de Educación, tomar decisiones frente a los mecanismos de evaluación, por lo tanto, se hace necesaria una caracterización de éstos.

Dentro del abordaje investigativo se encontraron estudios nacionales e internacionales que permitieron comprender tanto el tema como el problema planteado. 
Zambrano, R. (2006) realizó una tesis doctoral titulada La evaluación formativa en la enseñanza aprendizaje del inglés. El propósito de la investigación fue abordar de manera explícita todo lo relacionado con la evaluación y los diferentes tipos de evaluación que existen, resaltando la evaluación formativa. Igualmente destaca la evaluación del proceso de enseñanza y aprendizaje, afirmando que esta no solo repercute en el aula de clase, sino también en otros aspectos. Este estudio orientó la presente investigación, ya que dice que un enfoque pedagógico debe ser integral y que complemente a los estudiantes en todas sus formas, es decir, social, afectiva y cognitiva.

Asimismo, la mencionada tesis doctoral mostró como resultado que una parte del profesorado realizaba una evaluación tradicional, otra parte evaluaciones mixtas y otra parte evaluación formativa; además que los procesos evaluativos formativos requieren sistematización, tiempo y esfuerzo de parte de los profesores como de los alumnos.

Rosales (2014) en su libro Evaluar es reflexionar sobre la enseñanza, dice que: Desde la perspectiva del profesor, evaluar es reflexionar sobre los procesos de aprendizaje de sus alumnos, sobre su propia actuación como docente, sobre las circunstancias institucionales y sociales que influyen en ellos. Esta reflexión tiene por objeto profundizar en el conocimiento de aquello que se evalúa y facilita su valoración, así como la adopción de medidas de perfeccionamiento (p.37).

La evaluación forma parte esencial a la hora de analizar el aprendizaje de cada estudiante; ésta se articula en tres fases: La recolección de datos, la valoración de los mismos y la toma de decisiones; esto con el fin de facilitar el proceso y permitir que la evaluación cumpla sus finalidades dentro del proceso pedagógico.
Rosales (2014) en su libro Criterios para una evaluación formativa: objetivos. Contenido. Profesor. Aprendizaje. Recursos, aborda "la problemática de la evaluación desde una perspectiva formativa, es decir, un instrumento que posibilita el perfeccionamiento del proceso didáctico y de cada uno de los componentes del mismo" (p. 11), esto teniendo en cuenta como factor principal el papel del docente frente al conocimiento, su planeación didáctica, la naturaleza de los contenidos y la metodología empleada, a fin de determinar cuáles son los resultados; de esa forma se permite reestructurar esos componentes y replantear la metodología utilizada y así ofrecer un mejor proceso de enseñanza-aprendizaje.

Por su parte, Vásquez, (2012) aborda el concepto de evaluación como parte integral de la vida, ya que continuamente nos estamos sometiendo a ella; sostiene que no pasa un día sin que al finalizar la jornada no se haga un balance general de lo que se realizó y cómo se hizo, qué falta por hacer; por tal razón, no es posible pensar y organizar un proceso de enseñanza sin este componente.

Asimismo, la evaluación forma parte del proceso educativo y toda información que se obtenga de ella debe orientarse a la mejora del mismo. La evaluación formativa, identifica problemas, muestra soluciones y detecta los obstáculos a superar, además ayuda a perfeccionar el proceso educativo.

Los modelos evaluativos son el eje principal de las prácticas evaluativas en el campo educativo, Tyler (1950) fue el pionero en desarrollar el primer modelo evaluativo en los años treinta, titulado "modelo de evaluación orientado a objetivos", (p.102) para este autor, el verdadero aprendizaje es aquel que transforma el comportamiento del ser humano, su manera de pensar, sentir y reaccionar a ciertas situaciones. Igualmente, el autor resalta la importancia del currículo y afirma que las fuentes importantes 
para su diseño son "el sujeto, la sociedad y los contenidos" (102), asimismo, considera los objetivos, las actividades, los recursos y el modo de evaluación como parte fundamental de este modelo, porque por medio de ellos se determina el éxito o el fracaso del mismo.

Lo anterior propicia el planteamiento de la pregunta y los objetivos de la investigación.

¿De qué manera los modelos evaluativos de aula de los docentes de instituciones educativas públicas y privadas de la ciudad de Tuluá se encuentran en concordancia con las políticas de calidad educativa nacional?

\section{GENERAL}

Caracterizar los Modelos Evaluativos de aula de los docentes de instituciones educativas públicas y privadas de la ciudad de Tuluá en relación con los índices de calidad Educativa.

\section{ESPECÍ́FICOS}

> Caracterizar la concepción sobre evaluación desde la legislación educativa colombiana.

Caracterizar la concepción sobre evaluación en el aula de clase desde la propuesta de legislación educativa nacional.

Identificar los modelos evaluativos de los docentes de tres instituciones educativas públicas y tres privadas de la ciudad de Tuluá.

$>$ Contrastar los modelos evaluativos de aula con la concepción evaluativa nacional.

Contrastar los modelos evaluativos de aula de cada institución con respecto al cumplimiento las políticas de calidad educativa nacional propuestas.

\section{REVISIÓN DE LA LITERATURA}

Es importante destacar los diferentes estudios e investigaciones previas que se encontraron en este proceso investigativo y que contribuyen a esta construcción teórica y conceptual, además se pretende esclarecer su influencia para este proyecto.

El primer postulado tiene como título las relaciones entre el SIEE, PEI y la evaluación en el aula: análisis de caso en la Institución Educativa Oliverio Lara Borrero de la ciudad de Neiva, (Trujillo, 2015) el cual dice que hay poca articulación entre los proyectos educativos institucionales, los sistemas de evaluación educativa y la práctica pedagógica docente, los resultados obtenidos mostraron que los docentes, directivos y toda la comunidad educativa conocen los diferentes tipos de evaluación sus concepciones, acercamientos a su definición y comprensión de la evaluación pero que al momento de llevarla al aula de clase no se hacía lo planeado y lo dicho y terminaba convirtiéndose en un proceso sumativo. De la misma manera era importante relacionar el currículo con el contexto social y cultural, de tal manera que haya correlación entre los objetivos y los resultados. No obstante, es importante ver que se resalta la formación integral de los estudiantes en la normativa vigente, el PEI y el SIEE, por lo que así mismo tiene que ser la evaluación, incluyente y diversa. El estudio desarrolló un análisis que consistió en comparar información a partir de las entrevistas y encuestas, las cuales fueron fundamentales para el acercamiento a las diferentes definiciones que se tenían sobre evaluación y cómo se llevaban a cabo. Dicho lo anterior, estos postulados toman como punto de partida la poca relación existente entre tres componentes que son fundamentales en la educación colombiana, el PEI el SIEE y la pedagogía docente, ya sea debido a los resultados de las pruebas ICFES o simplemente por el hecho de conocer lo que 
pasa en las instituciones educativas respecto a la evaluación. Este artículo le aporta a nuestro trabajo ya que de allí hemos podido usar las Evaluaciones Educativas que América Latina necesita (Ravela, y otros, 2008) la cual exalta la importancia de le evaluación educativa, los cambios que ha mostrado la evaluación y muchas incógnitas que allí mismo se resuelven como: ¿Qué grados y qué disciplinas es importante evaluar? ¿Cada cuánto tiempo es necesario y adecuado realizar las evaluaciones? ¿Para qué se quiere evaluar? ¿Quiénes usarán los resultados y con qué propósitos? ¿Qué se espera saber que no se sepa ya? Entre otras preguntas y respuestas que aportaron para conocer a profundidad lo que con evaluación educativa se refiere.

Del mismo modo se encontró el trabajo titulado Practicas evaluativas de los docentes y sistema institucional de evaluación de estudiantes en el instituto integrado Francisco Serrano del municipio San Juan de Girón (Ramirez \& Rodriguez, 2014) este trabajo cuenta con un proceso de recolección y análisis de las practicas evaluativas de los docentes y los sistemas de evaluación de estudiantes esto con el fin de relacionarlas y conocer si hay una sinergia entre estas. Lo principal a estudiar en este proceso principalmente fue la Ley 115 de educación y el decreto 1290 el cual es guía para la evaluación en Colombia. La población y muestra en este trabajo fueron los grados 5 to y 9 no del instituto integrado Francisco Serrano del municipio San Juan de Girón ya que en estos grados se presentan simulacros de las pruebas ICFES, se realizó una encuesta a 36 docentes y las entrevistas realizadas se hicieron a 6 docentes y 2 estudiantes. Las conclusiones de este trabajo muestran que hay un amplio conocimiento teórico sobre le evaluación en el aula, que es procesual, no es sumativa solamente sino que es sistemática y de retroalimentación constante, pero al momento de revisar, observar y analizar las practicas evaluativas dieron cuenta que se estaban retomando las practicas evaluativas tradicionales, o sea que los estudiantes eran sujetos pasivos y el docente era el único agente evaluador en este proceso de aprendizaje. En adición a esto, lo importante para nuestro trabajo es poder adaptar algunas de las preguntas de la entrevista realizada a los docentes para poder cumplir con los objetivos propuestos, además de esto nos sirve también la investigación titulada "La otra cara de la evaluación" Pino (2006) puesto que allí de abarca el cambio que ha tenido la evaluación a través del tiempo, de cómo ha pasado de ser una evaluación tradicional y pasiva a una más interactiva, y donde el estudiante es un ser participativo capaz de evaluarse así mismo, además de esto se determina que los docentes deben capacitarse específicamente en evaluación para poder hacer un buen uso de ella.

Asimismo se tiene el artículo titulado el concepto de calidad educativa: una mirada crítica desde el enfoque históricocultural (Rodríguez, 2010) En donde se plantea que el concepto de calidad educativa se ha visto transformada con el tiempo además de que se entiende de una manera descentralizada a lo que debería ser, pues su concepto se deriva de una lógica empresarial tal como de donde proviene el término de la evaluación. Este artículo investigativo no cuenta con procesos experimentales o estudios de caso, solo es un recorrido que se le hace a la historia detrás de la calidad educativa con el fin de dar a conocer como se ha tergiversado el concepto y además plasmarlo de manera clara al contexto actual. Del mismo modo, el autor cita diferentes postulados que proponen y dicen que la calidad educativa se ha ido transformando de manera constructivista dejando de lado que esta se debe ver como un negocio donde la calidad se mide por números o que hay algún evaluador de la calidad, sino que la calidad educativa tiene que verse como un fin que el estudiante debe alcanzar para cumplir con el estándar (Rodríguez, 2010) "en el mundo de los negocios 
la calidad se define desde la satisfacción del cliente o del consumidor, pero en el mundo de la educación debe definirse desde los fines que persigue". Igualmente el autor también da a entender que los estudiantes son seres humanos, que se motivan o desmotivan, que tienen necesidades y deseos como cualquier persona, volviendo así el concepto de calidad educativa al contexto sociocultural de la época en que la se encuentre, por eso no se puede hablar de calidad educativa de hace 100 años a la calidad educativa del hoy, tal como el autor sigue la idea vygotskiana "de que el cambio y el desarrollo de las ideas, la aparición y la muerte de los conceptos puede explicarse si se relacionan con el sustrato sociocultural de su época". Este artículo nutre de forma satisfactoria el concepto de calidad educativa que ha enriquecido conceptual y teóricamente este trabajo que también usó el concepto de calidad educativa, por esta razón es uno de los postulados que se ha escogido para que pudiese alimentar este estudio.

\section{METODOLOGÍA}

La metodología propuesta para el desarrollo de este proyecto de investigación se enmarcó en el enfoque cualitativo, dado que su propósito fue examinar la forma en que los individuos perciben y experimentan los fenómenos que los rodean, profundizando en sus puntos de vista, interpretaciones y significados (Punch, 2014; Lichtman, 2013; Morse, 2012; Encyclopedia of Educational Psychology, 2008; Lahman y Geist, 2008; Carey, 2007, y DeLyser, 2006). El objetivo de esta investigación fue realizar un acercamiento interpretativo del concepto de evaluación, partiendo de la revisión de los documentos propuestos por el Ministerio de Educación Nacional, en contraste con los supuestos teóricos del concepto de evaluación, contrastar la información obtenida de los documentos con las prácticas evaluativas de los docentes, en las instituciones objeto de estudio.

Una vez planteado el enfoque. La presente investigación se sirvió del paradigma interpretativo, el cual se caracteriza por ser una metodología flexible, que utiliza datos cualitativos provenientes de documentos, así como entrevistas y sesiones de enfoque. Igualmente, la investigación se desarrolló en un contexto natural, es decir, en contacto permanente con las instituciones y sus participantes, los docentes y estudiantes. El contexto de investigación son los docentes de educación media y media técnica de tres instituciones educativas del sector público, las cuales son: Institución Educativa Técnico de Occidente, Institución Educativa Técnico Industrial "Carlos Sarmiento Lora" y la Institución Educativa Julia Restrepo y tres del sector privado, las cuales son: colegio Nazareth, el Colegio Bilingüe Hispanoamericano y el colegio San Juan Bosco. Del total de docentes como universo de estudio, se tomó una muestra representativa para llevar a cabo los grupos de enfoque. Las instituciones educativas han sido escogidas con base en el puntaje obtenido en las pruebas Saber 11. Este indicador permitió reconocer que dichas instituciones se encuentran en mayor concordancia con índices de calidad educativa. De ahí que se hizo necesario indagar sobre las formas en cómo se concibió y se desarrolló la evaluación en cada institución y se revisó en qué medida estas se relacionaron con lo propuesto en documentos nacionales que ofrecen los entes gestores y evaluadores de la calidad educativa. Los resultados obtenidos generaron una discusión frente a los procesos, mecanismos $y$ herramientas que utilizan estas instituciones y de qué forma estas se convirtieron en un referente de procedimientos para otras instituciones del municipio.

El procedimiento para analizar los datos se hizo desde un enfoque de análisis inductivo, 
profundizando en los detalles para descubrir interrelaciones que se extraen de los datos cualitativos. Las categorías de análisis para la interpretación de los resultados de la revisión documental se generaron a partir de los lineamientos de índices de calidad y la teoría sobre evaluación existente. Igualmente, los criterios de revisión de Proyectos Educativos Institucionales se obtuvieron de los postulados de cada modelo pedagógico que posea la institución.

Las técnicas de recolección de datos fueron: Matriz para revisión de documentos nacionales sobre evaluación. Matriz para revisión de modelo evaluativo concebido en el PEI, una entrevista inicial a docentes y finalmente una matriz para interpretación de resultados.

\section{INSTRUMENTOS}

\section{REVISIÓN DOCUMENTAL.}

La revisión de los documentos se hizo para recoger y conocer la información necesaria sobre la evaluación en el aula de acuerdo a la normativa vigente, los SIEE y los PEI institucionales de cada colegio o institución (Valencia, s.f) Dice que "La revisión documental permite identificar las investigaciones elaboradas con anterioridad... hacer relaciones entre trabajos; rastrear preguntas y objetivos de investigación; observar las estéticas de los procedimientos (metodologías de abordaje); establecer semejanzas y diferencias entre los trabajos".

\section{MATRIZ METODOLÓGICA.}

(Rivas, 2015) Establece que "La matriz metodológica es el instrumento científico que permite hacer congruente y coherente el proceso de la medición de variables independientes, creando un marco de comparación racional y ordenada para la construcción de un cuestionario". Tal como lo plantea el autor, las matrices son cuadros en donde se organizan las categorías a analizar, es decir que se desmenuzan de manera concisa y clara los conceptos de manera entendible y lógica. Asimismo para este trabajo investigativo se realizaron diferentes matrices para revisar los documentos, primero se hizo una matriz de los decretos y la ley 115 de 1994 para saber lo que planteaban sobre evaluación, después se hizo una matriz sobre lo que decían los lineamientos sobre la evaluación y por último una matriz sobre lo que planteaba el SIEE y el PEI de los colegios a estudiar.

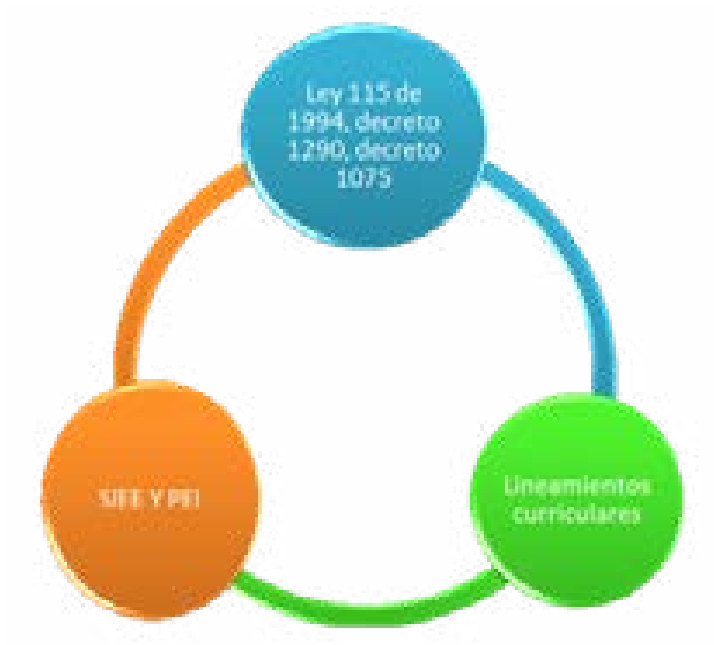

\section{ENTREVISTA SEMIESTRUCTURADA}

Para conocer las concepciones sobre evaluación de los docentes de las instituciones, se realizó una entrevista semiestructurada, (Baptista, Fernandez, \& Hernandez, 2014)lo definen "como una reunión para conversar e intercambiar información entre una persona (el entrevistador) y otra (el entrevistado) u otras (entrevistados)" Por eso, se optó por seleccionar la entrevista como técnica de recolección de la información porque ofrece una visión más amplia sobre lo que conocen los docentes como evaluación.

Preguntas para la entrevista.

- ¿Se ha formado específicamente en la evaluación del aprendizaje?

- ¿En qué se fundamenta principalmente su práctica evaluativa? 
- ¿Cuál es o son los tipos de evaluación del aprendizaje que emplea en el aula?

- ¿Qué criterios toma en consideración a la hora de evaluar el aprendizaje de los estudiantes?

- ¿Cree usted que los instrumentos y procedimientos de evaluación empleados en sus clases obtienen el resultado que espera en el aprendizaje de los estudiantes? ¿Por qué?

- ¿Existe un momento idóneo para hacer evaluación de los aprendizajes? De ser así descríbalo.

- ¿Qué entiende por evaluación?

\section{ANÁLISIS Y DISCUSIÓN}

Paraelanálisisy discusión del trabajo, las técnicas aplicadas en el tratamiento de la información consistió fundamentalmente en la codificación abierta, ya que esta permite que se ordene de manera clara todos los datos, además de que protege la ética de los docentes participantes ya que no se van a mencionar sus nombres si no que se van a usar códigos para referirse a ellos. La codificación permitió organizar la información por códigos y categorías y descartar la información irrelevante, la codificación abierta debido a su flexibilidad permite agrupar las categorías de análisis, comparar si hay algunas parecidas o que se puede agrupas en la misma categoría estos autores (Gonzalez, Hernandez, \& Hernandez, 2006) Dicen que "Usamos la codificación para comenzar a revelar significados potenciales y desarrollar ideas, conceptos e hipótesis; vamos comprendiendo lo que sucede con los datos (empezamos a generar un sentido de entendimiento respecto al planteamiento del problema)." Es por eso que la codificación se hizo esencial para realizar la respectiva categorización de la información y esto favoreció la construcción del núcleo del trabajo para interpretar, comparar los datos y poder darles sentido, para luego mostrar lo más relevante y de la misma manera obtener resultados más fáciles de mostrar.

A continuación se presentan los resultados de la codificación con su respectiva gráfica.

Los resultados obtenidos por esta gráfica indican que el desarrollo del aprendizaje integral del estudiante para seguimiento a las competencias y niveles de desempeño y la Identificación de las estrategias pedagógicas para el desempeño del estudiante son los propósitos más frecuentes hallados en los modelos evaluativos de 6 instituciones educativas de Tuluá, ya que ambas representan el $31 \%$ cada una, de la frecuencia mostrada, que el análisis del saber y las características de aprendizaje de los estudiantes representa el $25 \%$ de la frecuencia y por último está la gestión y evaluación para el logro de metas y estrategias con el $13 \%$ indicando que es la que menos frecuencia posee.

De acuerdo con lo anterior es importante destacar que los resultados de la evaluación permanente realizada por las Instituciones y la forma como la han afrontado ha buscado identificar, apreciar y estimar cómo va el desarrollo de los estudiantes a partir de los procesos pedagógicos y administrativos implementados con miras a elevar la calidad educativa. Sin embrago, se requiere analizar con mayor detalle los resultados obtenidos en cada categoría. Es así como el porcentaje de $13 \%$ en Gestión y evaluación para el logro de metas y estrategias, nos convoca a revisar los objetos de desarrollo establecidos en cuanto a lo deseable en el proceso educativo desde el Horizonte Institucional del PEI.

Es importante que, para elevar el porcentaje en esta categoría se tenga presente las necesidades y expectativas de la comunidad sus intereses, las relaciones que se construyen entre las gestiones, las aspiraciones de sus miembros, los procesos administrativos y los factores del 
contexto que posibiliten la dinamización de las estrategias.

Es necesario recordar que el artículo 80 de la Ley General de Educación debe evaluar "la calidad de la enseñanza que se imparte, el desempeño profesional del docente y de los docentes directivos, los logros de los alumnos, la eficacia de los métodos pedagógicos, de los textos y materiales empleados, de la organización administrativa y física de las instituciones educativas y la eficiencia de la prestación del servicio".

Hoy, más que nunca, se debe concretar y desarrollar un modelo de gestión Institucional orientado hacia todos los niveles con una visión estratégica y Pedagógica transformadora para que, desde diferentes escenarios, se promuevan estrategias inteligentes que den paso a resultados educativos de calidad que den razón, sentido, significado y fundamento a la educación.

En cuanto a la categoría "analizar el saber y las características de aprendizaje de los estudiantes", de la cual se obtuvo un porcentaje del $25 \%$, nos lleva al desafío de indagar por las diferentes formas que se han establecido frente a las formas dadas en el ensañar y aprender. En este sentido se requiere aproximarnos a la validez del conocimiento adquirido, los fundamentos filosóficos, teóricos e ideológicos sobre el cual se ha soportado el proceso formativo. Indudablemente el porcentaje obtenido no se presenta como uno de los mejores, sin embargo nos reta a investigar sobre la naturaleza del conocimiento de los estudiantes.

Es interesante analizar las teorías pedagógicas con las cuales se ha orientado el conocimiento, las mediaciones didácticas, el aprendizaje situado y la relación social que genera todo el ambiente escolar, visto todo esto desde la propia psicología del aprendizaje y la epistemología educativa.
La construcción del conocimiento y el saber propio incorporado al sistema educativo es pues una súper estructura que hace del proceso de aprendizaje de los estudiantes un constructo solido con el cual están en la capacidad de desarrollar competencias con las cuales se puedan enfrentar de manera eficaz y eficiente a la sociedad mundo tan desafiante del siglo XXI.

“En Pedagogía un conocimiento en educación es válido si sirve para educar (tiene significación, metodológicamente hablando, y por eso se le atribuyen propiedades de válido y fiable al conocimiento)" (Touriñán, 2014; Peters, 1979; Pring, 2014).

De otra parte, si bien es cierto que la aproximación al saber y características de aprendizaje se dan socialmente, es también cierto que la autonomía frente al conocimiento responde a las necesidades y expectativas de cada estudiante y el uso que pretende darle a sus conocimientos. Los estudiantes de acuerdo con sus condiciones propias generan motivaciones internas y externas, según el nivel que pretende o desea alcanzar.

"Cada persona ha de comprometerse con su propia formación. Ha de querer aprender de forma independiente y autónoma en contextos cambiantes. Ha de querer ser competente para evaluar y tomar decisiones sobre qué, cuándo, cómo necesita aprender. El interés y motivación resultan claves para un aprendizaje eficaz. Cuando el conocimiento resulta relevante y significativo para la persona, tanto intelectual como afectivamente, es asimilado de forma más fácil, duradera y eficaz" (Csikszentmihalyi, 1993; Seligman, 1991).

Otra categoría de análisis que nos convoca a una profunda reflexión es el "desarrollo del aprendizaje integral del estudiante para seguimiento a las competencias y niveles de desempeño". El porcentaje alcanzado fue de $31 \%$ y nos sitúa en términos matemáticos en una 
tercera parte de ese desarrollo integral deseado. Un dato no menor, toda vez que, cuando hablamos de "desarrollo del aprendizaje integral", estamos haciendo referencia a la formación del ser humano en totalidad, el desarrollo humano que tanto se promueve en los escenarios educativos en su PEI, específicamente en su modelo pedagógico. Si bien es cierto el aprendizaje es social, es igualmente cierto que los estudiantes desarrollan unas competencias que van paulatinamente cumpliendo unos niveles de desempeño esperados.

La sociedad del conocimiento y la información de la actualidad nos obligan a asumir nuestros estudiantes como seres que potencialmente son constructores, innovadores y transformadores desde perspectivas que exijan evolución cognitiva, estructura mental, desarrollo de las diferentes inteligencias, construcción de sistemas complejos de aprendizaje, desarrollo de valores, proyectos personales de vida y sociabilidad, y es aquí donde la escuela se convierte en el territorio que posibilita la transformación y generación de cambio al formar estudiantes que puedan intervenir en diferentes contextos a partir del desarrollo de sus competencias. El desarrollo del aprendizaje integral obliga a aprender día a día, a un aprendizaje permanente, riguroso, con estrategias eficientes que respondan a la solución de problemas e intervención en situaciones relevantes del contexto y del conocimiento mismo.

En este sentido, la denominada "educación situada", siempre ha pretendido vincular la escuela con la vida. "Según Díaz Barriga, una premisa importante a considerar en la educación situada, es que el aprender, hacer y reflexionar son acciones indisociables. Este tipo de enseñanza, puede fortalecer la identidad de las personas y prepararlas para la vida en sociedad, y permitirían que tanto el currículo como la instrucción misma de los estudiantes se centraran en los aprendizajes que resultan significativos tanto para su desempeño en el mundo exterior como en lo relativo a lo que acontece en la comunidad escolar misma". DíazBarriga A. F. s/f. Enseñanza situada: vínculo entre la escuela y la vida. Mc Graw Hill, México, Cap. 1-5, pp. 1-163

Cada persona desarrolla competencias a lo largo de su vida para darle sentido a la misma de ahí que es muy importante saber pensar y actuar.

\section{Para ello es importante:}

"hacerlo desde criterios razonables y susceptibles de crítica; ser sensible a las exigencias cambiantes de los contextos; desarrollar el pensamiento reflexivo, crítico y creativo. Los aprendizajes necesarios en la sociedad del conocimiento, como gran reto para nuestro tiempo, se han de construir sobre los siguientes pilares: A) Aprender a conocer, B) Aprender a querer y sentir, C) Aprender a hacer, D) Aprender a convivir, E) Aprender a ser,F) Aprender sobre el conocer, el querer, el sentir". (Delors, 1996; García García, 2006).

"La educación debe permitir que todos puedan aprovechar la información, recabarla, seleccionarla, ordenarla, manejarla y utilizarla.

Por consiguiente, la educación tiene que adaptarse en todo momento a los cambios de la sociedad, sin por ello dejar de transmitir el saber adquirido, los principios y los frutos de la experiencia".

Declaración mundial sobre la educación superior en el siglo XXI: visión y acción. (1998). http:// www.crue.org/dfunesco.htm [15/02/06]

"Es necesario educar para vivir y convivir con lo mejor del ser humano, no sólo proporcionar instrucción para desempeñarse en un trabajo. Aunque lo anterior pueda ser considerado como un ideal o una utopía, el tenerlo en mente y el trabajar con esa orientación, dará 
resultados cada día más positivos. Una manera de iniciar el paso de la teoría a la acción, es procurar que en la práctica, los sistemas educativos proporcionen a las personas, el conocimiento y los recursos necesarios para que establezcan su propio "plan de vida", desarrollen sus capacidades integrales $y$ mediante su interacción con la sociedad, ésta se dirija hacia mejores horizontes".

Delors J. 1996. La Educación encierra un tesoro. UNESCO, Comisión Internacional sobre la educación para el siglo XXI. SantillanaUNESCO.

Frente a las competencias el Ministerio de Educación Nacional de Colombia se ha centrado en una formación pertinente y de calidad. Este presupuesto apunta al desarrollo integral de los estudiantes, el conocimiento y formación no se sitúa en la adquisición únicamente de información sino en procesos significativos con los cuales los estudiantes sean proactivos y productivos; sin embargo para llegar a esta meta se necesita una trasformación curricular muy profunda que transforme la práctica docente aplicando modelos y teorías pedagógicas que se adecuen a esta necesidad sentida en el contexto glocal de la educación. Los resultados en pruebas estandarizadas no son sino un indicador que permite evaluar el proceso como tal.

"Todos los estudiantes, independientemente de su procedencia, situación social, económica y cultural, cuenten con oportunidades para adquirir conocimientos, desarrollar las competencias y valores necesarios para vivir, convivir, ser productivos y seguir aprendiendo a lo largo de la vida". (MEN Revolución Educativa 2002-2010)

En la actualidad el Ministerio de Educación Nacional y el ICFES (Ley 1324 de 2009), direccionan toda la política de calidad de la educación en Colombia, que va desde la generación de los documentos ministeriales como tal, (lineamientos curriculares, estándares de competencia, derechos básicos de aprendizaje (DBA), mallas curriculares y de referencia, orientaciones pedagógicas y varios programas que le apuntan al mejoramiento de la calidad educativa; así mismo el ICFES, establece guías orientadoras y programas con los cuales los estudiantes se aproximan con mayor precisión a lo que es una prueba estandarizada).

No cabe duda que toda esta información se convierte en motivo de análisis puesto que los resultados tendrían que ser mejores a los obtenidos.

La cuarta categoría de la primera gráfica, (propósitos de los sistemas), Identificación de las estrategias pedagógicas para el desempeño del estudiante, nos arroja un porcentaje también del $31 \%$. Indudablemente este resultado es una alerta, puesto que guarda una relación directa con el encuentro docente-estudiante, y parece indicar en una primera instancia que el trayecto formativo posiblemente la estrategia no ha cumplido su misión. Desde lo pedagógico es sabido que la estrategia es el vehículo por el cual se moviliza el conocimiento de forma metodológica integrando y contextualizando el currículo, para la obtención de unos resultados deseables por parte del estudiante y de la Institución.

"El proceso de enseñar a pensar; enseñar a aprender; enseñar a ser y estar. Esto implica el desarrollo de diversas estrategias, que incluyen el fomento de la creatividad, el sentido de responsabilidad, el fomento de la independencia en la búsqueda del conocimiento, la incentivación de un acercamiento interdisciplinario hacia el saber y la posibilidad del desarrollo de las aspiraciones individuales. Asimismo con la flexibilidad curricular se propone disminuir el tiempo de las clases presenciales, para que el estudiante realice otras actividades (culturales, deportivas, recreativas, etc.) que le permitan formarse integralmente, aprendiendo a cumplir 
un reglamento, aprendiendo a ganar, a perder, a competir, a ser tolerante, a trabajar en grupo, a resolver conflictos" (González, 2006, p. 3).

Es preciso expresar que la estrategia pedagógica trae consigo un método y una técnica para la aplicación de dichos métodos. La práctica pedagógica no se puede limitar a una sola estrategia de ahí que el docente debe experimentar y aplicar diversas estrategias con las cuales los estudiantes accedan al conocimiento.

La estrategia se concibe como "una secuencia de actividades que el profesor decide como pauta de intervención en el aula" (Rodríguez, 1994, p. 168). Según Rodríguez, una estrategia de enseñanza se puede convertir en un método de enseñanza si se dan las siguientes condiciones: 1. Un alto nivel de rigor en sus planteamientos, es decir, se fundamenta en planteamientos científicos. 2. Una fuerte experimentación previa. 3. Una difusión y formalización suficiente (Rodríguez, 1994).

Los procesos de aprendizaje y el uso de estrategias requieren del desarrollo de operaciones mentales que den significado a la actividad realizada por el.

"la educación escolar promueve el desarrollo en la medida en que genera la actividad mental constructiva del alumno responsable haciendo de él una persona única, irrepetible en el contexto, de un grupo social determinado" (Coll, (2007, p. 15).

Las estrategias pedagógicas, según Bravo (2008) "constituyen los escenarios curriculares de organización de las actividades formativas y de la interacción del proceso enseñanza aprendizaje donde se alcanzan conocimientos, valores, prácticas, procedimientos y problemas propios del campo de formación" (Bravo, 2008, p. 34), considerándose para ello fundamental, una fuerte formación teórica en los docentes quienes, orientarán la calidad de los procesos de enseñanza aprendizaje.

En esta gráfica se pueden apreciar los fines $u$ objetivos de los sistemas de evaluación de 6 instituciones educativas de la ciudad de Tuluá, asimismo se puede ver que el seguimiento, control y medición del proceso de aprendizaje con el fin de orientarlo en una valoración especifica de acuerdo con sus intereses, es quien lleva la ventaja en frecuencia con el $40 \%$ de veces en que este se repite, seguido por la aplicación de estrategias de valoración integral para los desempeños de los estudiantes con el $20 \%$, después está, flexible, interpretativa, participativa , formativa con el $20 \%$ también, y por último, conocer el proceso de desarrollo del estudiante en diferentes campos de su desempeño con el $20 \%$ restante.

La categoría de seguimiento, control y medición del proceso de aprendizaje, necesariamente necesita de analizar las características propias tanto del proceso de aprendizaje como de la evaluación. La concepción actual de la educación plantea una revisión continua de los procesos que permita apreciar el progreso y las dificultades presentadas en el trayecto formativo, con el fin de gestionar planes de mejoramiento que aborden de manera integral a los estudiantes en sus dimensiones. Así mismo, se requiere revisar los principios pedagógicos y metodológicos con los cuales se ha orientado el conocimiento.

En palabras de lucio (1989) para saber "cómo se educa hay que conocer cómo es el hombre, cómo crece: la pedagogía se apoya en la sicología y en la sicología evolutiva. Para saber cómo se enseña hay que saber cómo se aprende: la didáctica se apoya en la sicología del aprendizaje" (Lucio, 1989, p. 42).

Es de anotar, que es de suma relevancia en esta categoría, hacer seguimiento y control a los diferentes ritmos de aprendizaje, capacidades, 
contexto y significado que le dan a su propio proceso de formación.

En el acto didáctico la motivación en el aula favorece el rendimiento académico del estudiantado, donde el espacio influye en el significado que el estudiante le brinda a su proceso de enseñanza aprendizaje, los recursos de trabajo y una buena propuesta de contenidos, potencia las posibilidades cognitivas de los educandos (Montoya , 1997).

En la actualidad muchos países alrededor del mundo, están poniendo en práctica una variedad de estrategias destinadas a mejorar la calidad de la educación y en este orden de ideas el seguimiento, control y medición, proporcionan información validad para mejorar la prestación de los servicios educativos.

Finalmente, es muy importante que las necesidades, expectativas e intereses de los estudiantes estén incorporadas al currículo y en concordancia con las políticas nacionales e internacionales en materia de educación.

"La Organización para la Cooperación y el Desarrollo Económico señala la importancia de la preparación de los jóvenes en la sociedad contemporánea y de la facultad de las personas para intervenir con amplio criterio en la definición de las políticas públicas que repercutan e influyan en su vida personal, social, profesional y cultural (ocde2007)".

Frente a las estrategias de valoración integral de los desempeños de los estudiantes, de nuevo nos dan una alerta dado que el porcentaje obtenido de $20 \%$, indican que en el proceso se presenta alguna falencia y que es necesario revisar la misma.

De ahí que, es preciso sugerir la aplicación de estrategias motivantes desde el aula de clase que generen encuentros realmente significativos y de formación integral con las que se evidencie el protagonismo del educando. Es muy importante adecuar la estrategia al contexto local, regional, nacional e internacional.

La orealc-unesco (2007) se refiere también a la importancia de que los contenidos de la educación sean significativos para personas de distintos estratos sociales, culturas e intereses; de forma tal que pueda aprehenderse la cultura local, nacional y mundial, para constituirse como sujetos universales, con autonomía, autogobierno, libertad e identidad. La pertinencia, demanda la inclusión del 'otro', pese a las diferencias culturales, de acceso al conocimiento, al aprendizaje, al éxito escolar y a las oportunidades sociales.

De acuerdo con el planteamiento anterior, y en concordancia con los resultados anteriores, se requiere sentar el proceso evaluativo $y$ formativo sobre bases teóricas, conceptuales y metodológicas con un enfoque holístico, donde la práctica cotidiana del saber genere un cambio de mentalidad, que conduzca a una renovación de los sistemas con los cuales se ha direccionado el acto educativo y centrarlo en métodos activos que articule el currículo y la vida misma del estudiante en contexto.

Para Harris (2002) y Hopkins (2000), el éxito escolar reside en lo que sucede en el aula, de ahí que la forma en que se organizan las experiencias de aprendizaje pueden marcar la diferencia en los resultados de los alumnos en relación con su desarrollo cognitivo y socioafectivo. Rodríguez (2009) coincide en que, independientemente de las variables contextuales, las formas y los estilos de enseñanza del profesor y su gestión en el aula son aspectos decisivos a considerarse en el logro de los resultados, y se hacen evidentes en la planeación didáctica, en la calidad de las producciones de los estudiantes y en la calidad de la autoevaluación de la práctica docente, entre otras. 
En esta gráfica se puede apreciar, la importancia que representa el desempeño superior, el desempeño alto, el desempeño básico y el desempeño bajo en los indicadores de desempeño con una ventaja del $71 \%$, mientras que, enfatizan la integración, estructuración, síntesis y aplicación de conocimientos, saberes, actitudes y valores está representado por el $29 \%$ restante.

Los indicadores necesariamente nos acercan a la meta deseada o planteada de ahí cobra importancia analizar con profundidad que indicadores se han establecido en el proceso de planeación y evaluación, y así determinar en qué fase del proceso se encuentran los resultados alcanzados. Al ser los indicadores señales precisas, es relevante revisar cada indicador y así realizar el plan de mejora. Los indicadores nos permiten hacer comparaciones y elaborar juicios del desempeño del proceso de forma cualitativa y cuantitativa. Los indicadores y los niveles de desempeño dan al establecimiento educativo una categorización a nivel nacional de ahí la importancia que estos representan.

Los indicadores deben ser considerados elementos correspondidos y articulados dentro de un Sistema Nacional de Indicadores, estos pueden compararse para obtener una mejor dimensión de las circunstancias que lo afectan; su lectura y análisis dependen de la forma como se agrupen y relacionen, para así construir herramientas que permitan monitorear la evolución de un tema específico, realizar comparaciones y analizar en forma detallada de la situación. (MEN, Sistema Nacional de Indicadores - Colombia 2013)

El sistema de indicadores pretende evaluar el rendimiento del sistema de educación colombiano, antes que comparar las instituciones que lo componen o las entidades territoriales. Sin embargo, es indudable que diversas características importantes del desarrollo, del funcionamiento y del impacto del sistema educativo, solo puedan ser evaluadas mediante un análisis de los resultados educativos y de su relación con los recursos utilizados y los procesos a un nivel individual e institucional.

La estructura de un Sistema de Indicadores Educativos para el caso Colombiano busca describir la situación actual de la educación en Colombia, el estado de los procesos educativos que se han desarrollado y el impacto que estos han tenido en cuanto a cobertura, calidad, acceso a la educación, entre otros.

La gráfica de criterios de evaluación muestra la relevancia que posee, tener en cuenta todas las dimensiones del desarrollo del estudiante y de sus avances en las instituciones ya que cuenta con el $60 \%$ de la frecuencia, mientras que los demás aspectos como; conocer el estado del desarrollo integral del educando y de sus avances cuenta con el $13 \%$ de la frecuencia, utilizar apoyos y herramientas técnicas y tecnológicas, acorde a las necesidades de los estudiantes está representado con el $13 \%$ de la frecuencia y por último tener en cuenta el desarrollo cognitivo, personal y social, al evaluar y valorar cada una también cuenta con el $13 \%$ de la frecuencia mostrada.

En conclusión se puede dilucidar que hay grandes diferencias en los resultados de los datos recogidos ya que como se pudo observar son diferencias bastantes enmarcadas que poco a poco van mostrando sus efectos en los índices de calidad educativa.

Indudablemente los resultados de este grafica nos convoca a una profunda reflexión y análisis, puesto que, recoge unas categorías fundamentales centradas en el estudiante, sus necesidades, intereses, expectativas, inteligencias, desarrollo cognitivo, dimensiones y la forma como recibe o apropia las herramientas y/o estrategias didácticas establecidas por el docente. 
La significación de una formación integral va más allá del aspecto académico e involucra el aprendizaje y el conocimiento del ser, así como el aprendizaje y la enseñanza en la formación de principios y valores.

"La educación es integral en la medida en que enfoque a la persona al estudiante como una totalidad y no lo considera únicamente en su potencial cognoscitivo o en su capacidad para el quehacer técnico o profesional" (Orozco, 2007, p. 142).

Es importante así mismo, identificar los elementos dentro del plan de estudio o dentro de la política educativa de la institución que aporta a la integralidad y formación de los estudiantes.

Se debe analizar las ventajas y desventajas del diseño curricular, las asignaturas en cuanto a su objeto de conocimiento y los instrumentos metodológicos y didácticos dispuestos para el trayecto formativo por parte del docente.

Los criterios de evaluación apuntan a la calidad educativa y por ende a la formación integral contribuyendo en el rendimiento, la formación en valores, el trabajo en grupo y el desarrollo de competencias.

Ninguna forma de desarrollo puede ser expresado sin su fundamental relación con nuestro desarrollo y evolución biológica, porque en tal caso, sea cual sea la explicación, ninguna definición del desarrollo de lo vivo tendría sentido. Esto es, y quiere decir que el desarrollo existe y es válido únicamente cuando es garante de la vida, tanto individual como colectiva. Este es el primer punto de referencia para esta "Teoría del Desarrollo Integral”. Gotemburgo, noviembre 2006. Julio Alberto Rodríguez

De otra parte en la educación actual el uso de herramientas tecnológicas es un apoyo fundamental para el alcance de las competencias y adquisición de conocimiento. Estas herramientas, estimulan, motivan, sensibilizan, despiertan la creatividad, ingenio y curiosidad por explorar diferentes escenarios del conocimiento. No se puede negar la fuerte influencia de estos en la movilización de las operaciones mentales y desarrollo de las inteligencias.

Las nuevas tendencias de la Educación buscan mayor participación del alumnado en el proceso educativo, así como un aprendizaje significativo y colaborativo, esto hace que las TIC se conviertan en medios didácticos imprescindibles, de ahí que la UNESCO (2016) avizora que emergerán nuevos entornos pedagógicos. Ello supone crear servicios de educación a distancia y disponer de tecnologías que no solo reduzcan los límites del tiempo y el espacio, sino que también establezcan sistemas de enseñanza con calidad, favoreciendo un mayor acceso a la escolaridad y la democratización del aprendizaje (Quesada, 2014).

Para los nativos digitales resulta más fácil comprender los códigos que se emplean en cada una de plataformas e-learning, en tanto desde niños se han familiarizado con las herramientas tecnológicas, además de que su preparación se ha especializado a medida que en la escuela se ha orientado y formado el modo de usar esos instrumentos. Solo el escaso nivel de acceso a las TIC, que persiste en algunas familias y escuelas, constituye en la actualidad un obstáculo para que los jóvenes mantengan elevados perfiles en el empleo de las TIC, y específicamente de las plataformas elearning.

DOI: dx.doi.org/10.21556/edutec.2018.65.1067

De acuerdo con las teorías actuales de la psicología cognitiva, la información por sí misma no propicia conocimiento, es necesario proveer una serie de condiciones que favorezcan el proceso de aprendizaje. No se trata simplemente de entregar información para que el aprendizaje se produzca. Es necesario propiciar las "transacciones didácticas fundamentales que 
se presentan entre docentes y estudiantes o estudiantes entre sí, y que contribuyen a la circularidad comunicativa indiscutible en la construcción de los saberes", Fainholc (1999, pp. $64-65)$.

De acuerdo con lo anterior aproximar el uso de herramientas y medios masivos de comunicación, al proceso educativo permite un desarrollo cognitivo no solo individual sino social, pues el requiere de la aplicación de estrategias cooperativas y colaborativas que contribuyan con la calidad educativa.

Es preciso aunar esfuerzos y emprender nuevas investigaciones que ayuden a entender el nuevo y complejo mundo pedagógico generado por las TIC, marcado por la dificultad añadida de ser un ámbito tremendamente cambiante debido a la rapidez con la que crecen y evolucionan las nuevas tecnologías. Las TIC, en sus diferentes formatos, versiones yutilidades, han conquistado, por derecho propio, un protagonismo en la vida cotidiana de gran parte de la población, sobre todo, la juvenil. Sus profundas implicaciones y su fuerte impacto sociológico es tan inmenso que aún no existen suficientes estudios para medirlo en toda su intensidad (Cózar y De Moya, 2013). Los componentes de la presente sociedad del conocimiento precisan un saber, un uso y un dominio adecuados para gestionar debidamente las habilidades propias de la era digital, tanto para desenvolverse en el momento presente como para capacitarse con vistas al futuro. Es decir, tanto para el estudio de hoy como para el trabajo de mañana, los alumnos deben conocer sus capacidades tecnológicas, con sus fortalezas y debilidades, siendo el contexto escolar el espacio adecuado para aprender a ello y alcanzar una mínima competencia digital (Herrera, 2015).

\section{REFERENCIAS BIBLIOGRÁFICAS}

Baptista, P., Fernandez, C., \& Hernandez, R. (2014). Metodología de la investigación sexta edición. México: mcgraw-HILL / interamericana editores, s.a. de c.v.

Gonzalez, M., Hernandez, A., \& Hernandez, I. (2006). El constructivismo en la evaluación de los aprendizajes del álgebra lineal. Educere, 14.

Ramirez, G., \& Rodriguez, A. (2014). Practicas evaluativas de los docentes y sistema institucional de evaluación de estudiantes en el instituto integrado francisco serrano del municipio san juan de girón. Ibagué: practicas evaluativas de los docentes y sistema institucional de evaluación de estudiantes en el Instituto Integrado Francisco Serrano del Municipio San Juan De Girón.

Ravela, P., Arregui, P., Valverde, G., Wolfe, R., Ferrer, G., Martínez, P., y otros. (2008). Las evaluaciones educativas que américa latina necesit. Revista Iberoamerica de Evaluación Educativa, 19.

Rivas, A. (2015). Capítulo 11, contrucción de la matriz metodológica. ResearchGate, 16.

Rodríguez, A. (2010). El concepto de calidad educativa: una mirada crítica desde el enfoque históricocultural. "Actualidades Investigativas en Educación”, 30.

Trujillo, C. (2015). Las relaciones entre SIEE, PEI y la evaluación en el aula: Análisis de cas en la Institución Educativa Oliverio Lara Borrero de la ciudad de Neiva. journalusco. 
Valencia, V. (s.f). Revisión documental en el proceso de investigación. Universidad tecnológica de Pereira, 5.

Rosales, Carlos. Criterios para una evaluación formativa . Madrid: Narcea, 2014.

Rosales, M. Proceso evaluativo: evaluación sumativa, evaluación Formativa $y$ Assessment su impacto en la educación actual. Buenos Aires, Argentina., 2014.

Tyler, R. Basic principles of currículum and instruction. Chicago: University of Chicago Press, 1950.

Vaccarini. La evaluación de los aprendizajes en la escuela secundaria actual. Buenos Aires: Facultad de Desarrollo e Investigación Educativos, 2014.

Vasquez, B. Evaluación Formativa. Chiapas, México: Tapachula, 2012.

Zambrano, G. La evaluación formativa en la enseñanza-aprendizaje de Inglés. URV. Tarragona Tesis doctoral inédita. , 2006. 